\title{
Pseudocarbynes: Charge-stabilized Carbon Chains
}

\author{
Pilarisetty Tarakeshwar, ${ }^{, \dagger}$ Peter R. Buseck, ${ }^{\dagger, \ddagger}$ Harold W. Kroto ${ }^{\S}$ \\ ${ }^{\dagger}$ School of Molecular Sciences, Arizona State University, Tempe, Arizona 85287-1604, United \\ States \\ School of Earth and Space Exploration, Arizona State University, Tempe, Arizona 85287-6004, \\ United States \\ ${ }^{\S}$ Department of Chemistry and Biochemistry, Florida State University, Tallahassee, Florida \\ 32306-4390, United States
}

\section{Supporting Information}

Figure S1. Comparison of the calculated B3LYP/cc-pVDZ and CCSD(T)/cc-pVDZ vibrational frequencies of the $\mathrm{C}_{6}$ carbon chains in (a) singlet states and (b) triplet states.

Figure S2: Calculated (B3LYP/cc-pVDZ) Raman spectra of an isolated $\mathrm{C}_{10} \mathrm{H}_{2}$ polyyne and its complexes with several sizes of gold clusters. Except for some minor shifts, the peaks associated with both the $\mathrm{C} \equiv \mathrm{C}$ and $\mathrm{C}-\mathrm{C}$ stretches appear at similar frequencies in all the complexes. The multiple peaks around $2100 \mathrm{~cm}^{-1}$ coalesce into a single peak as the size of the gold cluster increases.

Figure S3. Comparison of the calculated (a) BP86/def2-TZVP, and (b) B3LYP/cc-pVDZ, Raman spectra of the $\mathrm{C}_{10} \mathrm{H}_{2}-\mathrm{Au}_{8}$ and $\mathrm{C}_{10} \mathrm{H}_{2}-\mathrm{Au}_{12}$ complexes.

Figure S4. Calculated absorption spectra of different $\mathrm{C}_{10}$ species in both gas-phase and methanol solvent obtained from TDDFT(B3LYP/cc-pVDZ) calculations. The polarizable continuum model (PCM) model was used to represent the methanol solvent. The absorption maxima of all the systems are red-shifted in methanol.

Figure S5. Optimized $2 \times 2 \times 2$ structure of a hypothetical crystal of carbon chains intercalated with $\mathrm{Au}_{8}$ clusters. The unit cells of this pseudocarbyne crystal is indicated by light black lines.

\footnotetext{
*tarakesh@asu.edu
} 


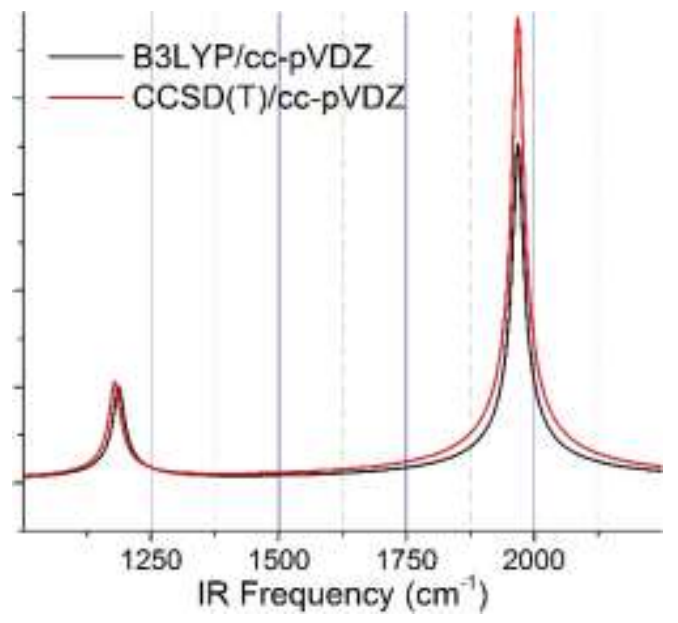

(a)

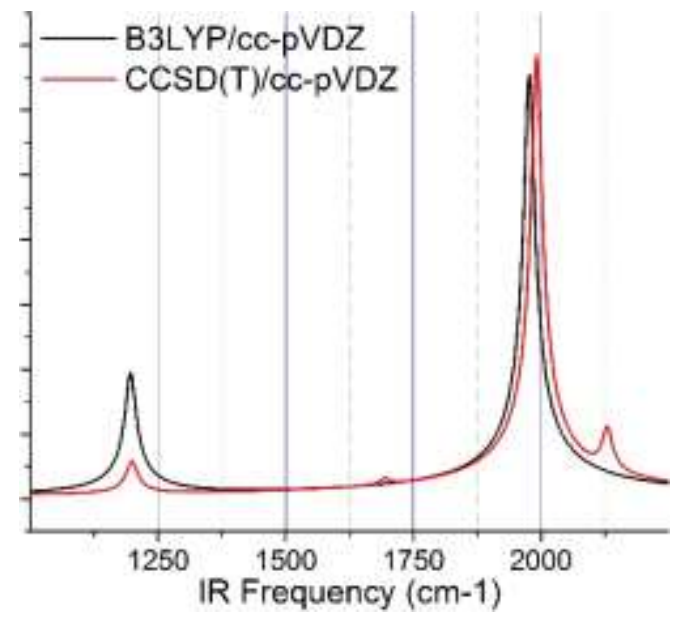

(b)

Figure S1. Comparison of the calculated B3LYP/cc-pVDZ and CCSD(T)/cc-pVDZ vibrational frequencies of the $\mathrm{C}_{6}$ carbon chains in (a) singlet states and (b) triplet states. 


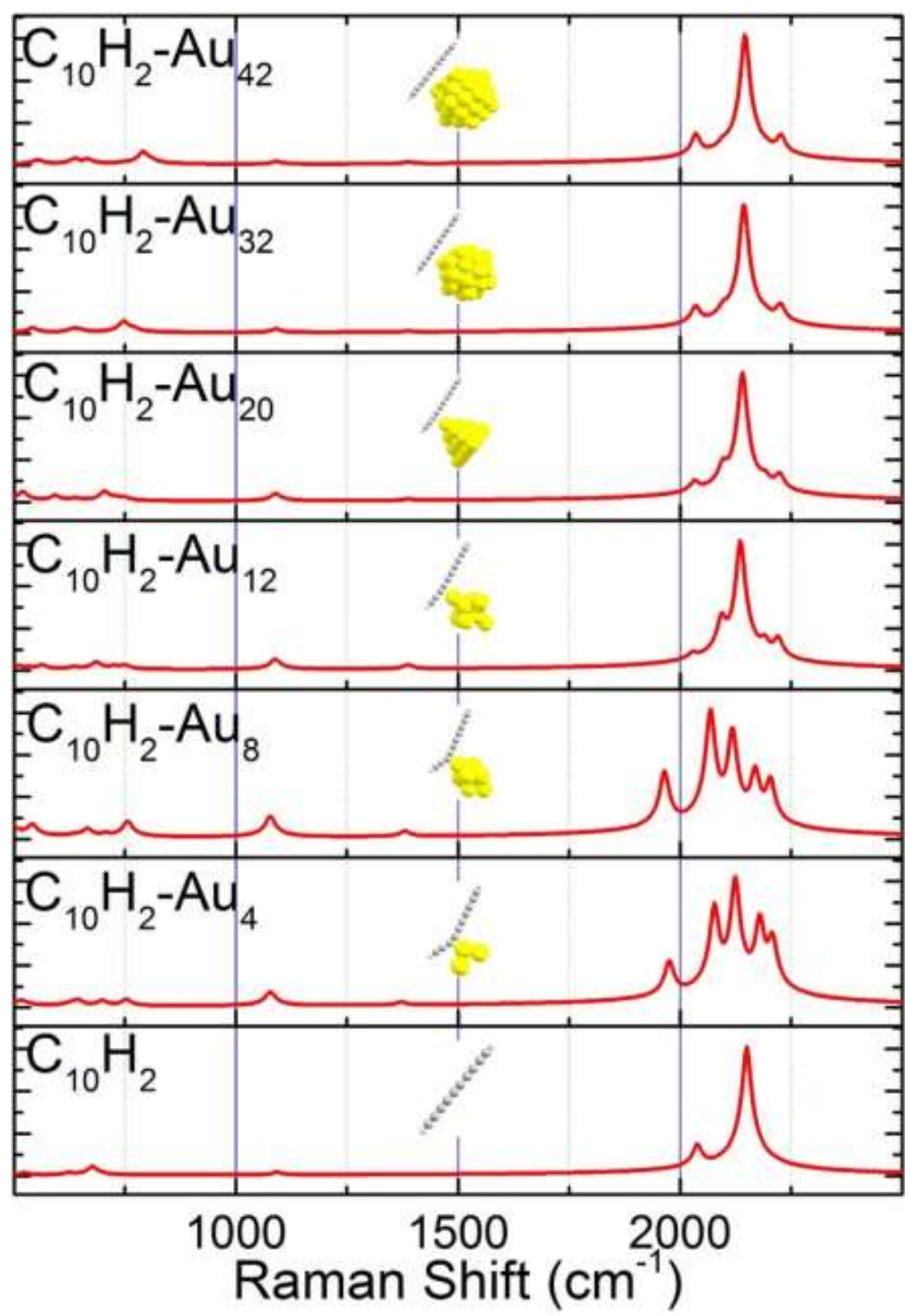

Figure S2: Calculated (B3LYP/cc-pVDZ) Raman spectra of an isolated $\mathrm{C}_{10} \mathrm{H}_{2}$ polyyne and its complexes with several sizes of gold clusters. Except for some minor shifts, the peaks associated with both the $\mathrm{C} \equiv \mathrm{C}$ and $\mathrm{C}-\mathrm{C}$ stretches appear at similar frequencies in all the complexes. The multiple peaks around $2100 \mathrm{~cm}^{-1}$ coalesce into a single peak as the size of the gold cluster increases. 

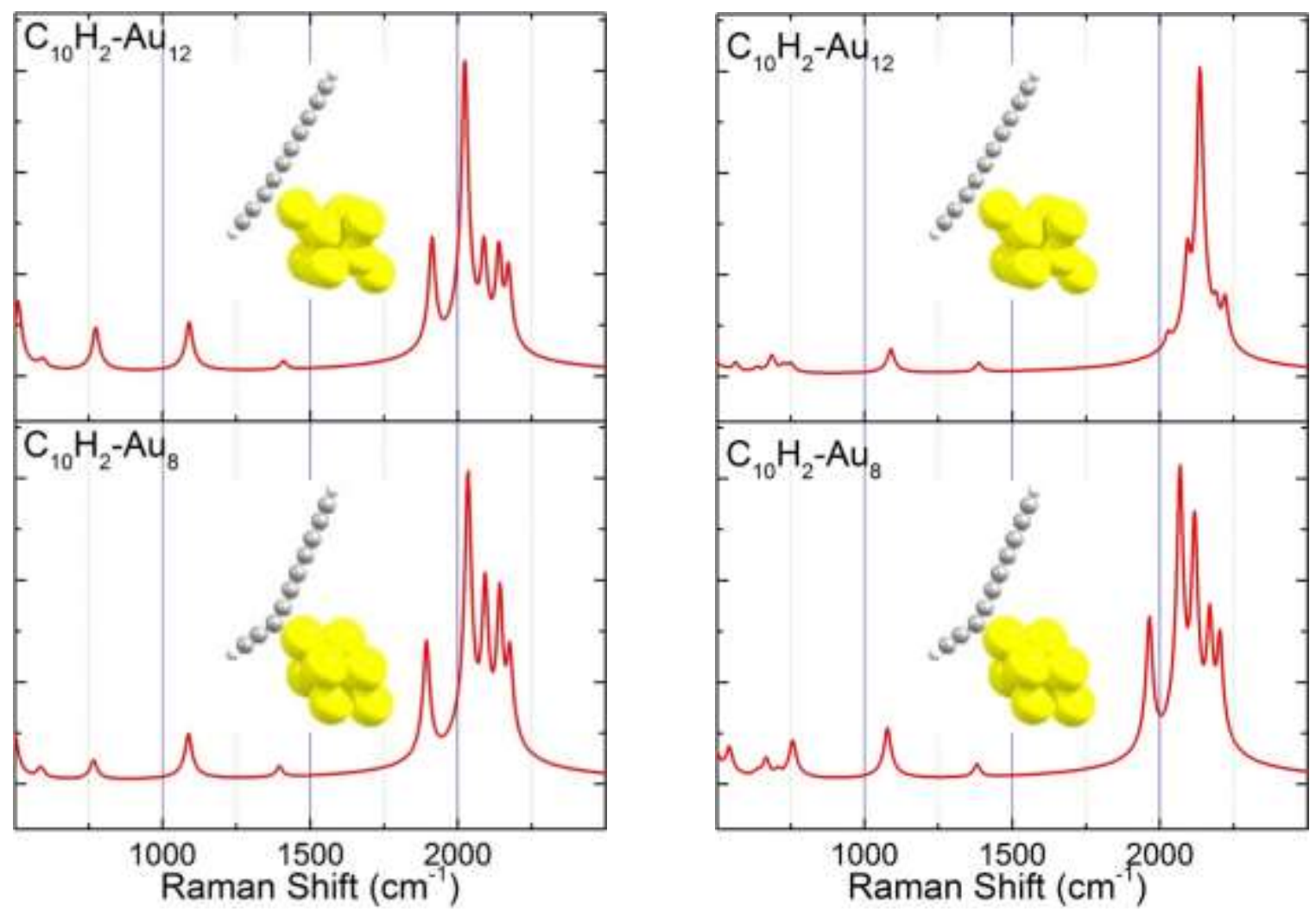

Figure S3. Comparison of the calculated (a) BP86/def2-TZVP, and (b) B3LYP/cc-pVDZ, Raman spectra of the $\mathrm{C}_{10} \mathrm{H}_{2}-\mathrm{Au}_{8}$ and $\mathrm{C}_{10} \mathrm{H}_{2}-\mathrm{Au}_{12}$ complexes. 


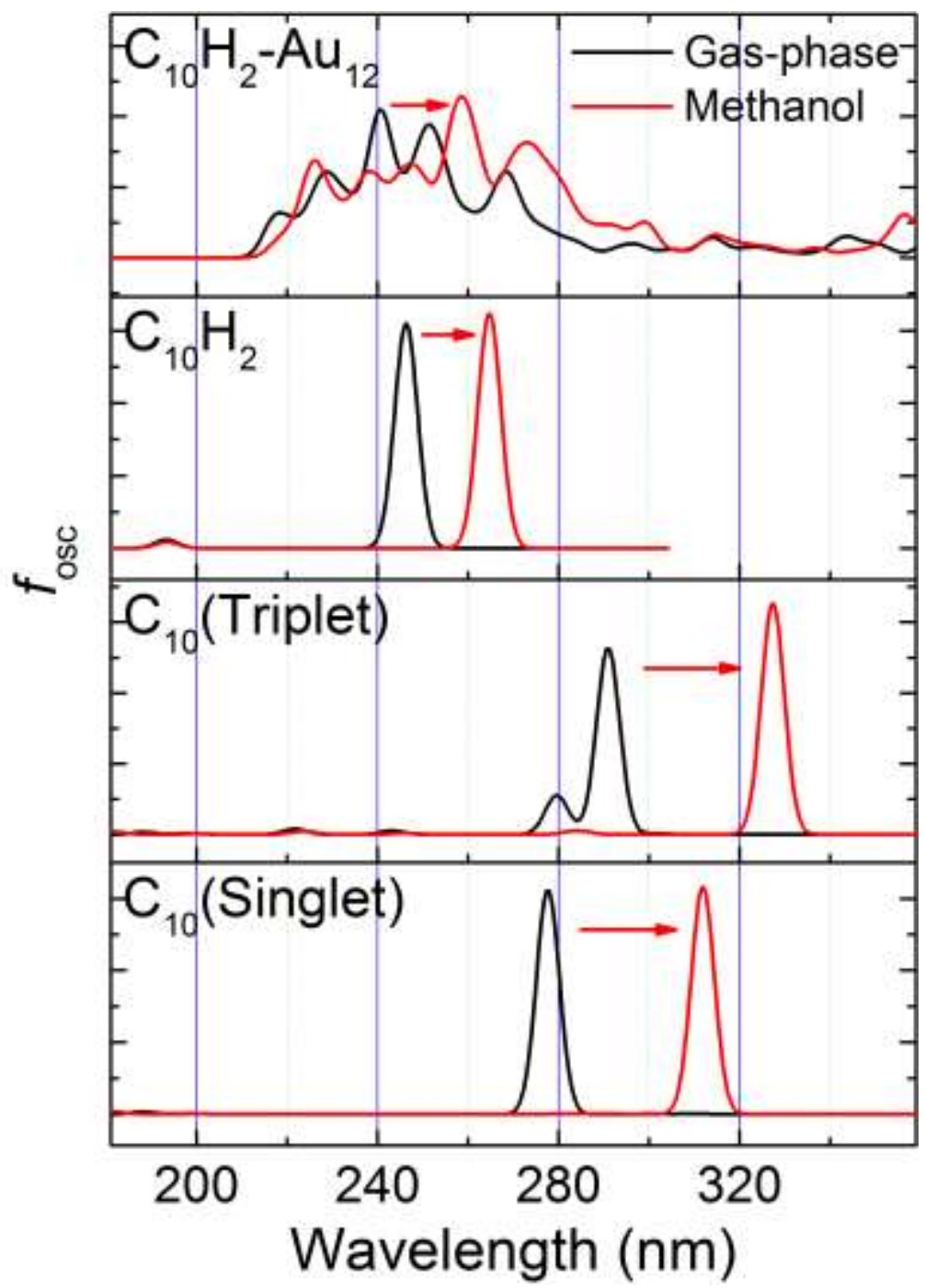

Figure S4. Calculated absorption spectra of different $\mathrm{C}_{10}$ species in both gas-phase and methanol solvent obtained from TDDFT(B3LYP/cc-pVDZ) calculations. The polarizable continuum model (PCM) model was used to represent the methanol solvent. The absorption maxima of all the systems are red-shifted in methanol. 


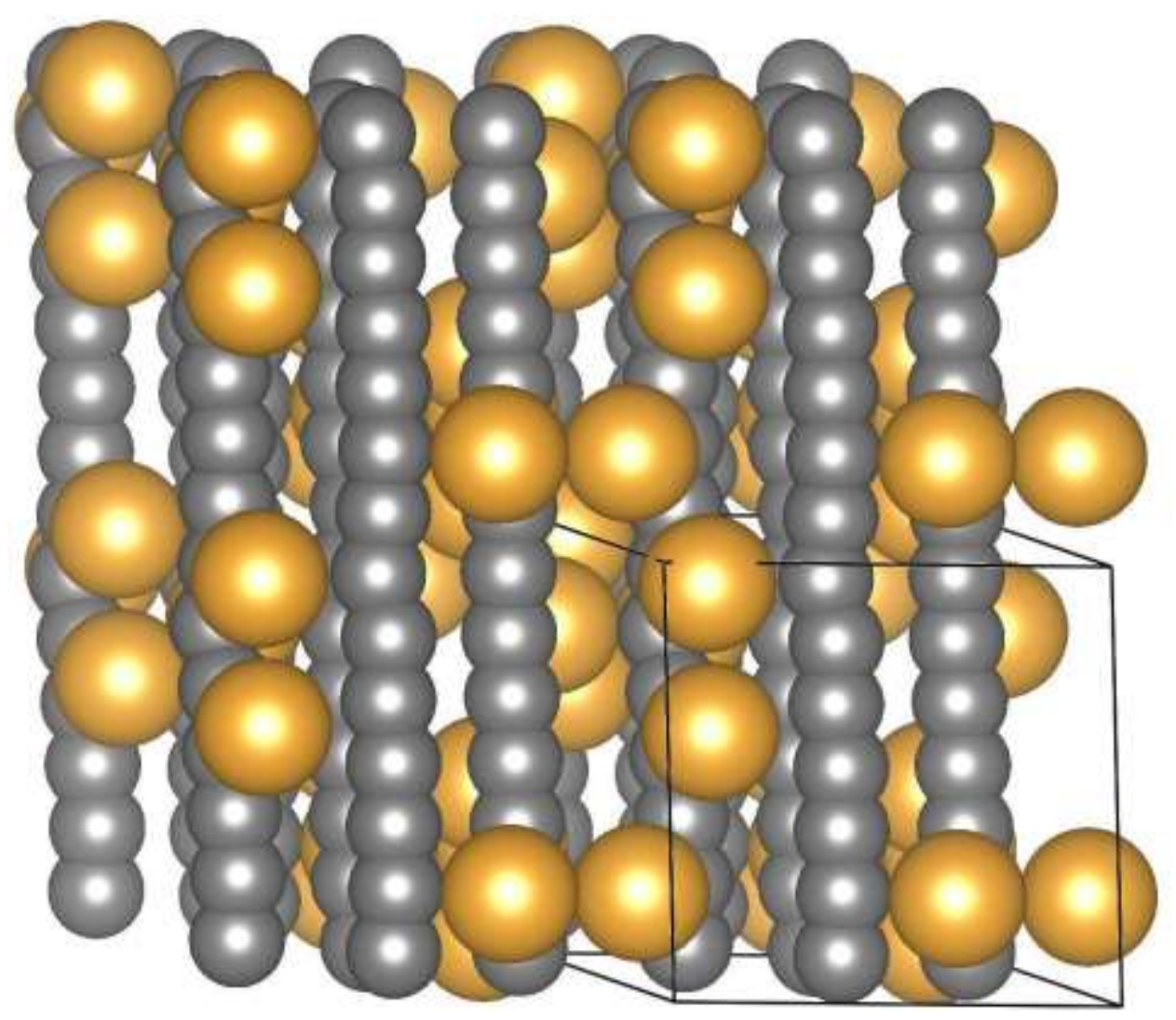

Figure S5. Optimized $2 \times 2 \times 2$ structure of a hypothetical crystal of carbon chains intercalated with $\mathrm{Au}_{8}$ clusters. The unit cells of this pseudocarbyne crystal is indicated by light black lines. 